\title{
Riemann Function and Methods of Group Analysis
}

\author{
Akimov Andrey ${ }^{1}$, Chernov Igor ${ }^{2}$, Abdullina Rufina ${ }^{3}$ \\ 453103, Sterlitamak, Russia, Lenina street 47A \\ Department of Physics and Mathematics, Bashkir state university Sterlitamak branch
}

\begin{abstract}
In this paper for the hyperbolic equation was constructed the four-parameter group and with the help of
\end{abstract} the group was found the solution of the Cauchy problem by the Riemann method for a hyperbolic equation

Keywords: Problem Cauchy, Riemann's Function, Hyperbolic Equation, Group Analysis.

\section{INTRODUCTION}

The general solution of the Cauchy problem for a second-order linear hyperbolic partial differential equation in two variables is often given in terms of integrals involving an auxiliary solution called the Riemann function.

By either method, completion of the problem requires the determination of auxiliary function, which is a difficult task because there is no unified method for actually finding this function. Methods for finding Riemann functions have been given by Riemann [1], Copson [4], and Mackie [8]. Regrettably, there are still only a few equations with known Riemann functions.

This paper is of a synthetic nature, being a result of combining Riemann's method [3] for integrating second-order linear hyperbolic equations with Lie's classification [11] of such equations. Using the results for the group classification of homogeneous hyperbolic equation of the second order, it was suggested to find a function of Riemann using the symmetries of the equation.

\section{Preliminaries}

Let's consider the following hyperbolic equation of the second order:

$$
L v=v_{x x}-v_{y y}+\frac{\mu^{2}(1-\lambda) \lambda v}{4 \sin ^{2} \mu(2 x+\sigma)}=0,(0<\lambda<1, \sigma>0, \mu \in R)
$$

in an open domain $D$, which is bounded by curves of $A C(y+x=0), C B(x-y=1)$ and with the section $A B(x=-\sigma)$.

Let's pose the problem of Cauchy: Find in the domain $D$ function $u(x, y)$, satisfying the conditions

$$
\begin{aligned}
& v(x, y) \in C(\bar{D}) \cap C^{1}(D \cup A B) \cup C^{2}(D), \\
& \operatorname{Lv}(x, y) \equiv 0, \quad(x, y) \in D, \\
& \lim _{x \rightarrow-\sigma}(\operatorname{tg} \mu(x+y+\sigma)-\operatorname{tg} \mu(x-y+\sigma))^{\lambda-1} v(x, y)=\tau(y), \\
& \lim _{x \rightarrow-\sigma}(\operatorname{tg} \mu(x+y+\sigma)-\operatorname{tg} \mu(x-y+\sigma))^{\lambda} \frac{\partial v}{\partial x}=v(y),
\end{aligned}
$$

\footnotetext{
${ }^{1}$ Corresponding Author: andakm@ rambler.ru
} 
American Research Journal of Mathematics, Volume 1, Issue 3, 2015

ISSN 2378-704X

where $\tau(y), v(y)$ - given sufficiently smooth functions.

Applying real coordinate transformation $\xi=x+y, \eta=x-y$ equation (1) leads to the canonical form:

$u_{\xi \eta}+\frac{\mu^{2}(1-\lambda) \lambda u}{\sin ^{2} \mu(r+\sigma)}=0$,

where $r=\xi+\eta$ and boundary values

$$
\begin{aligned}
& \lim _{\xi+\eta \rightarrow-2 \sigma}(\operatorname{tg} \mu(\xi+\sigma)-\operatorname{tg} \mu(\eta+\sigma))^{\lambda-1} u(\xi, \eta)=\widetilde{\tau}(\xi) \\
& \lim _{\xi+\eta \rightarrow-2 \sigma}(\operatorname{tg} \mu(\xi+\sigma)-\operatorname{tg} \mu(\eta+\sigma))^{\lambda}\left(\frac{\partial u(\xi, \eta)}{\partial \eta}+\frac{\partial u(\xi, \eta)}{\partial \xi}\right)=\widetilde{v}(\xi) .
\end{aligned}
$$

We shall define the operator $L$ by the identity

$$
L u=u_{\xi \eta}+a(\xi, \eta) u_{\xi}+b(\xi, \eta) u_{\eta}+c(\xi, \eta) u
$$

The operator $L^{*}$ defined by the identity

$$
L^{*} v=v_{\xi \eta}-(a v)_{\xi}-(b v)_{\eta}+c v
$$

is known as the adjoint of $L$.

To solve the problem we use the method of Riemann, which is based on the following identity:

$$
2\left(v L u-u L^{*} v\right)=\left(v u_{\eta}-u v_{\eta}+2 a u v\right)_{\xi}+\left(v u_{\xi}-u v_{\xi}+2 b u v\right)_{\eta}
$$

If the functions $v$ and $u$ are such that $L u=L v=0$ throughout a domain $G$ bounded by sufficiently smooth closed curve $\Gamma$. Then an application of Green's theorem yields

$$
2 \iint_{G}\left(v L u-u L^{*} v\right) d \xi d \eta=\int_{\Gamma}\left[-\left(v u_{\xi}-u v_{\xi}+2 b u v\right) d \xi+\left(v u_{\eta}-u v_{\eta}+2 a u v\right) d \eta\right] .
$$

Riemann's method reduces the problem of integration of the equation

$$
L u=f \text {. }
$$

To that of constructing the auxiliary function $v=R\left(\xi, \eta ; \xi_{0}, \eta_{0}\right)$, solving the ad joint equation:

$L^{*} R=0$

and satisfying the following conditions on the characteristics:

$$
\begin{aligned}
& \left.\left(R_{\eta}-a R\right)\right|_{\xi=\xi_{0}}=0, \\
& \left.\left(R_{\xi}-b R\right)\right|_{\eta=\eta_{0}}=0, \\
& R\left(\xi_{0}, \eta_{0} ; \xi_{0}, \eta_{0}\right)=1 .
\end{aligned}
$$


American Research Journal of Mathematics, Volume 1, Issue 3, 2015

ISSN 2378-704X

Provided that the function $v$ is known, the solution of the Cauchy problem:

$$
L u=f,\left.u\right|_{\Gamma}=u_{0}(x),\left.\frac{\partial u}{\partial n}\right|_{\Gamma}=u_{1}(x)
$$

with data on an arbitrary non-characteristic curve $\Gamma$ is given by the formula

$$
\begin{aligned}
& u\left(\xi_{0}, \eta_{0}\right)=\frac{(u R)_{P}+(u R)_{Q}}{2}-\frac{1}{2} \int_{P Q}-\left(R u_{\xi}-u R_{\xi}+2 b u R\right) d \xi+ \\
& +\left(R u_{\eta}-u R_{\eta}+2 a u R\right) d \eta+\iint_{G} R f d \xi d \eta
\end{aligned}
$$

where the double integral is taken over the domain bounded by the characteristics $\xi=\xi_{0}, \eta=\eta_{0}$ and the curve $\Gamma$. The function $v=R\left(\xi, \eta ; \xi_{0}, \eta_{0}\right)$ is called Riemann's function, and the boundary-value problem (9) is called the characteristic Cauchy problem, or the Goursat problem. The solution of the Goursat problem is unique.

\section{MAIN RESUltS}

In our case, the equation adjoint equation (5) has the form

$u_{\xi \eta}+\frac{\mu^{2}(1-\lambda) \lambda}{\sin ^{2} \mu(r+\sigma)}=0$.

Let's note that in our case the desired function of Riemann $v=R\left(\xi, \eta ; \xi_{0}, \eta_{0}\right)$ satisfies the following conditions on the characteristics:

$$
\left.R\right|_{\eta=\eta_{0}}=\left.R\right|_{\xi=\xi_{0}}=1, \quad R\left(\xi_{0}, \eta_{0} ; \xi_{0}, \eta_{0}\right)=1
$$

The symmetry operator of the homogeneous equation (10) has the form [4]:

$$
X=v(\xi) \frac{\partial}{\partial \xi}+w(\eta) \frac{\partial}{\partial \eta}+\tau(\xi, \eta) u \frac{\partial}{\partial u} \text {. }
$$

Thus, as follows from [5], must be done the following relations:

$$
\begin{gathered}
\frac{\partial \tau}{\partial \xi}+\frac{\partial(b v)}{\partial \xi}+w \frac{\partial b}{\partial \eta}=0, \quad \frac{\partial \tau}{\partial \eta}+\frac{\partial(a w)}{\partial \eta}+v \frac{\partial a}{\partial \xi}=0, \\
\frac{\partial^{2} \tau}{\partial \xi \partial \eta}+a \frac{\partial \tau}{\partial \xi}+b \frac{\partial \tau}{\partial \eta}+\frac{\partial(c v)}{\partial \xi}+\frac{\partial(c w)}{\partial \eta}=0 .
\end{gathered}
$$

Substituting in this case $a=0, b=0, c=\frac{\lambda}{\sin ^{2} \mu(r+\sigma)}$, we'll obtain the following relations

$v_{\xi} \sin \mu(r+\sigma)-2 v \mu \cos \mu(r+\sigma)=$

$=-w_{\eta} \sin \mu(r+\sigma)+2 w \mu \cos \mu(r+\sigma)$.

The solution of this partial differential equation of the first order will functions 
American Research Journal of Mathematics, Volume 1, Issue 3, 2015

ISSN 2378-704X

$$
\begin{aligned}
& v=A \sin ^{2} \mu(\xi+\sigma)+B \sin \mu(\xi+\sigma) \cos \mu(\xi+\sigma)+C \cos ^{2} \mu(\xi+\sigma), \\
& w=-A \sin ^{2} \mu(\eta+\sigma)-B \sin \mu(\eta+\sigma) \cos \mu(\eta+\sigma)-C \cos ^{2} \mu(\eta+\sigma), \tau=D
\end{aligned}
$$

where $A, B, C, D$ - arbitrary constants. Thus, equation (12) admits the three-parameter group (in addition to stretchings of the variable $u$ and the infinite group consisting of addition to $u$ of any solution of the equation; this group is common to all linear equations) with the generators

$$
\begin{aligned}
& X_{1}=\sin ^{2} \mu(\xi+\sigma) \frac{\partial}{\partial \xi}-\sin ^{2} \mu(\eta+\sigma) \frac{\partial}{\partial \eta}, \\
& X_{2}=\sin \mu(\xi+\sigma) \cos \mu(\xi+\sigma) \frac{\partial}{\partial \xi}-\sin \mu(\eta+\sigma) \cos \mu(\eta+\sigma) \frac{\partial}{\partial \eta}, \\
& X_{1}=\cos ^{2} \mu(\xi+\sigma) \frac{\partial}{\partial \xi}-\cos ^{2} \mu(\eta+\sigma) \frac{\partial}{\partial \eta}, X_{4}=u \frac{\partial}{\partial u} .
\end{aligned}
$$

Let us find a linear combination of these operators,

$$
X=\alpha_{1} X_{1}+\alpha_{2} X_{2}+\alpha_{3} X_{3}+\alpha_{4} X_{4},
$$

where $\alpha_{1}, \alpha_{2}, \alpha_{3}, \alpha_{4}-$ arbitrary constants.

Following [6], let us require first the invariance of the characteristics $\xi=\xi_{0}, \quad \eta=\eta_{0}$. The invariance test has the form:

$$
X\left(\xi-\xi_{0}\right)=0, X\left(\eta-\eta_{0}\right)=0 .
$$

One can set $\alpha_{1}=1$, then obtain $\alpha_{2}=\operatorname{tg} \mu\left(\xi_{0}+\sigma\right), \alpha_{3}=\operatorname{tg} \mu\left(\eta_{0}+\sigma\right)$. One can readily verify that the resulting operator

$$
\begin{aligned}
& X=\left(\operatorname{tg} \mu\left(\xi_{0}+\sigma\right) \sin \mu(\xi+\sigma)-\cos \mu(\xi+\sigma)\right)\left(\operatorname{tg} \mu\left(\eta_{0}+\sigma\right) \sin \mu(\xi+\sigma)-\cos \mu(\xi+\sigma)\right) \frac{\partial}{\partial \xi}+ \\
& +\left(\operatorname{tg} \mu\left(\xi_{0}+\sigma\right) \sin \mu(\eta+\sigma)-\cos \mu(\eta+\sigma)\right)\left(\operatorname{tg} \mu\left(\eta_{0}+\sigma\right) \sin \mu(\eta+\sigma)-\cos \mu(\eta+\sigma)\right) \frac{\partial}{\partial \eta}
\end{aligned}
$$

is admitted by the Goursat problem (10).

Invariant of this operator have the form $I=\frac{\left(\operatorname{tg} \mu(\xi+\sigma)-\operatorname{tg} \mu\left(\xi_{0}+\sigma\right)\right)\left(\operatorname{tg} \mu(\eta+\sigma)-\operatorname{tg} \mu\left(\eta_{0}+\sigma\right)\right)}{\left(\operatorname{tg} \mu(\eta+\sigma)-\operatorname{tg} \mu\left(\xi_{0}+\sigma\right)\right)\left(\operatorname{tg} \mu(\xi+\sigma)-\operatorname{tg} \mu\left(\eta_{0}+\sigma\right)\right)}$. Now

if $f$ is assumed to be a function of $z=\frac{\left(\operatorname{tg} \mu(\xi+\sigma)-\operatorname{tg} \mu\left(\xi_{0}+\sigma\right)\right)\left(\operatorname{tg} \mu(\eta+\sigma)-\operatorname{tg} \mu\left(\eta_{0}+\sigma\right)\right)}{\left(\operatorname{tg} \mu(\eta+\sigma)-\operatorname{tg} \mu\left(\xi_{0}+\sigma\right)\right)\left(\operatorname{tg} \mu(\xi+\sigma)-\operatorname{tg} \mu\left(\eta_{0}+\sigma\right)\right)}$ the equation

(12) becomes

$$
z(1-z)^{2} f^{\prime \prime}(z)+(1-z)^{2} f^{\prime}(z)+(1-\lambda) \lambda f(z)=0 .
$$

With the change of variable $z=\frac{s}{s-1}$, obtain

$$
s(1-s) f^{\prime \prime}(s)+(1-2 s) f^{\prime}(s)-(1-\lambda) \lambda f(s)=0 .
$$


American Research Journal of Mathematics, Volume 1, Issue 3, 2015

ISSN 2378-704X

The solution of the obtained equation is function Gauss

$f=F(\lambda, 1-\lambda, 1 ; \mu)$.

Then the Riemann's function in the $(\xi, \eta)$ will have the form

$$
R\left(\xi, \eta, \xi_{0}, \eta_{0}\right)=F\left(\lambda, 1-\lambda, 1 ; \frac{\left(\operatorname{tg} \mu(\xi+\sigma)-\operatorname{tg} \mu\left(\xi_{0}+\sigma\right)\right)\left(\operatorname{tg} \mu(\eta+\sigma)-\operatorname{tg} \mu\left(\eta_{0}+\sigma\right)\right)}{\left(\operatorname{tg} \mu(\eta+\sigma)-\operatorname{tg} \mu\left(\xi_{0}+\sigma\right)\right)\left(\operatorname{tg} \mu(\xi+\sigma)-\operatorname{tg} \mu\left(\eta_{0}+\sigma\right)\right)}\right) .
$$

Substituting in the formula $a=0, b=0, f=0$ obtain

$$
\begin{aligned}
& u\left(\xi_{0}, \eta_{0}\right)=\frac{2 \beta \mu \Gamma(1-2 \beta)}{\Gamma^{2}(1-\beta)} \int_{-\eta_{0}-2 \sigma}^{\xi_{0}} \frac{\left(\operatorname{tg} \mu\left(\eta_{0}+\sigma\right)-\operatorname{tg} \mu\left(\xi_{0}+\sigma\right)\right)^{\lambda}\left(1+\operatorname{tg}^{2} \mu(t+\sigma)\right) \tilde{\tau}(t) d t}{\left.\left(\eta_{0}+\sigma\right)-\operatorname{tg} \mu(t+\sigma)\right)^{\lambda}\left(\operatorname{tg} \mu(t+\sigma)-\operatorname{tg} \mu\left(\xi_{0}+\sigma\right)\right)^{\lambda}}+ \\
& +\frac{2 \beta \mu \Gamma(1-2 \beta)}{\Gamma^{2}(1-\beta)} \int_{-\eta_{0}-2 \sigma}^{\xi_{0}} \frac{\left(\operatorname{tg} \mu\left(\eta_{0}+\sigma\right)-\operatorname{tg} \mu\left(\xi_{0}+\sigma\right)\right)^{\lambda} \widetilde{v}(t) d t}{\left(\operatorname{tg} \mu\left(\eta_{0}+\sigma\right)-\operatorname{tg} \mu(t+\sigma)\right)^{\lambda}\left(\operatorname{tg} \mu(t+\sigma)-\operatorname{tg} \mu\left(\xi_{0}+\sigma\right)\right)^{\lambda}} .
\end{aligned}
$$

Returning to the old variables $x$ and $y$, we'll get the solution of the Cauchy's problem

$$
\begin{aligned}
& u(x, y)=\frac{2 \beta \mu \Gamma(1-2 \beta)}{\Gamma^{2}(1-\beta)} \int_{y-x-2 \sigma}^{x+y} \frac{(\operatorname{tg} \mu(x-y+\sigma)-\operatorname{tg} \mu(x+y+\sigma))^{\lambda}\left(1+\operatorname{tg}^{2} \mu(t+\sigma)\right) \tilde{\tau}(t) d t}{(\operatorname{tg}-y+\sigma)-\operatorname{tg} \mu(t+\sigma))^{\lambda}(\operatorname{tg} \mu(t+\sigma)-\operatorname{tg} \mu(x+y+\sigma))^{\lambda}}+ \\
& +\frac{2 \beta \mu \Gamma(1-2 \beta)}{\Gamma^{2}(1-\beta)} \int_{y-x-2 \sigma}^{x+y} \frac{(\operatorname{tg} \mu(x-y+\sigma)-\operatorname{tg} \mu(x+y+\sigma))^{\lambda} \widetilde{v}(t) d t}{(\operatorname{tg} \mu(x-y+\sigma)-\operatorname{tg} \mu(t+\sigma))^{\lambda}(\operatorname{tg} \mu(t+\sigma)-\operatorname{tg} \mu(x+y+\sigma))^{\lambda}}
\end{aligned}
$$

THEOREM. If the functions $\tau(y) \in C^{2}\left[\frac{1}{2} ; 1\right] v(y) \in C^{2}\left[\frac{1}{2} ; 1\right]$, then the Cauchy's problem for equation (1) has a unique solution, which is defined by (14).

\section{REFERENCES}

[1] G. F. B. Riemann. Uber die Fortpflanzung ebener Luftwellen von endlicher Schwingungsweite. Abhandl. Konigl. Ges. Wiss. Gottingen, 8, (1860),43-65.

[2] Akimov A.A., On uniqueness Morawetz problem for the Chaplygin equation, IJPAM, vol. 97, No. 3, (2014), 369-375.

[3] Akimov A.A., Some Properties and Applications of the Riemann-Hadamard Function of Darboux Problem for Telegraph Equation, American Research Journal of Mathematics, Vol. 1, No. 3, (2015), 9-15.

[4] J.S. Papadakis广†, D.H. Wood, An addition formula for Riemann functions Journal of differential Equations, 24, (1977), 397-411.

[5] E.T. Copson, On the Riemann-Green Function, J. Rat.Mech. Anal., 1, (1958), 324-348.

[6] D.H.Wood, Simple Riemann functions, Bull. Amer. Math. Soc. vol. 82, No. 5, (1976), 737-739.

[7] M.M. Smirnov, Equations of mixed type, American Mathematical Society, United States, (1978).

[8] Courant R.,Hilbert D., Methods of Mathematical Physics, Interscience Publishers, Inc., New York, (1953).

[9] K.B. Sabitov and R.R. Il'yasov Ill-posedness of boundary value problems for one class of hyperbolic equations, Russian Mathematics(Iz. VUZ), vol. 45, No. 5, (2001), 56-60.

[10] A.G Mackie, Green's function and Riemann's method, Proc. Edinburgh Math. Soc., 14, (1964), 293-302

[11] Lerner, M.E., Qualitative Properties of the Riemann Function, Diff er. Uravn., vol. 27, No. 12, (1991), 21062120. 
American Research Journal of Mathematics, Volume 1, Issue 3, 2015

ISSN 2378-704X

[12] Bitsadze, A.V., Uravneniya matematicheskoi fiziki (Equations of Mathematical Physics), Moscow:Nauka, (1976).

[13] N. H. Ibragimov. Group analysis of ordinary differential equations and the invariance principle in mathematical physics (for the 150th anniversary of Sophus Lie). Uspekhi Mat. Nauk, 47, No. 4, (1992), 83144, . English transl., Russian Math. Surveys, 47(2) (1992), 89-156.

[14] P. J. Olver. Applications of Lie groups to diff erential equations. Springer-Verlag, New York, 1986. 2nd ed., (1993). 